Joint multi-user detection and intersymbol interference cancellation for WCDMA satellite UMTS - da ... Page 1 of 2

Let your

research do the talking.
Let Wiley Editing Services provide you with expert help to ensure your manuscript is ready for submission

Find out more and submit your manuscript

Research Article

\title{
Joint multi-user detection and intersymbol interference cancellation for WCDMA satellite UMTS
}

1. Mário Marques da Silva* and

2. Américo M. C. Correia

Article first published online: 28 JAN 2003

DOI: $10.1002 /$ sat.747

Copyright (C) 2003 John Wiley \& Sons, Ltd.

Issue

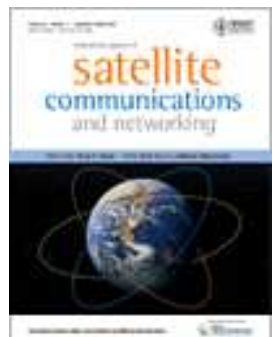

\section{International Journal of Satellite Communications and Networking}

Special Issue: Interference Suppression Techniques for Satellite Systems

Volume 21, Issue 1, (/doi/10.1002/sat.v21:1/issuetoc) pages 93-117, January/February 2003

Additional Information

\section{How to Cite}

da Silva, M. M. and Correia, A. M. C. (2003), Joint multi-user detection and intersymbol interference cancellation for WCDMA satellite UMTS. Int. J. Satell. Commun. Network., 21: 93-117. doi: 10.1002/sat.747

\section{Author Information}

Instituto de Telecomunicações, IST, Torre Norte 11.10 Av. Rovisco Pais, 1049-001 Lisboa, Portugal Email: Mário Marques da Silva (marquesilva@mail.telepac.pt)

*Instituto de Telecomunicações, IST, Torre Norte 11.10 Av. Rovisco Pais, 1049-001 Lisboa, Portugal

\section{Publication History}

1. Issue published online: 28 JAN 2003

2. Article first published online: 28 JAN 2003 
Joint multi-user detection and intersymbol interference cancellation for WCDMA satellite UMTS - da ... Page 2 of 2

3. Manuscript Received: 18 OCT 2002

- Abstract

- References (/doi/10.1002/sat.747/references)

- Cited By (/doi/10.1002/sat.747/citedby)

Get PDF (356K) (/doi/10.1002/sat.747/pdf)

\section{Keywords:}

S-UMTS; intersymbol interference; multiple access interference; commutation signaling; orthogonal code-di

\section{Abstract}

In this work we present two different intersymbol interference (ISI) cancellation systems, namely the commutation signaling (CS) and orthogonal codedivision transmit diversity (O-CDTD) in combination with satellite path diversity, which leads to a high diversity order. The ISI is due to the use of satellite path diversity (in order to introduce multipath diversity artificially). This work combines the proposed ISI cancellation systems with subtractive multi-user detectors - a variation of the conventional parallel interference cancellation (PIC) and successive interference cancellation (SIC) for the downlink channel of S-UMTS. The blind detection is also considered without knowledge of spreading sequences or the channel state information (CSI) of the interfering users with a noise whitening matched filter (NWMF) that combats the MAI through the whitening of the interfering power spectrum, also with and without the ISI cancellation systems - CS and O-CDTD. It is shown that the proposed PIC is a very promising detector to be used for the downlink of S-UMTS, especially when combined with CS or O-CDTD, the NWMF being efficient only when we consider jointly any of the proposed ISI cancellation systems for low number of interfering users. Copyright (C) 2003 John Wiley \& Sons, Ltd.

Get PDF (356K) (/doi/10.1002/sat.747/pdf)

\section{More content like this}

Find more content:

- $\underline{\text { like this article (/advanced/search/results? }}$ $\underline{\text { articleDoi }=10.1002 / \text { sat.747\&scope }=\text { allContent\&start }=1 \& \text { resultsPerPage }=20 \text { ) }}$

Find more content written by:

- Mário Marques da Silva (/advanced/search/results?searchRowCriteria[0].queryString="M\%C3\%A1rio Marques da Silva"\&searchRowCriteria[0].fieldName=author\&start=1\&resultsPerPage=20)

- Américo M. C. Correia (/advanced/search/results?searchRowCriteria[0].queryString="Am\%C3\%A9rico M. C. Correia"\&searchRowCriteria[0].fieldName $=$ author\&start $=1 \&$ resultsPerPage $=20$ )

- All Authors (/advanced/search/results?searchRowCriteria[0].queryString="M\%C3\%A1rio Marques da Silva" "Am\%C3\%A9rico M. C. Correia"\&searchRowCriteria [0].fieldName $=$ author\&start $=1 \&$ resultsPerPage $=20$ ) 